\title{
Angiomotin regulates endothelial cell migration during embryonic angiogenesis
}

\author{
Karin Aase, ${ }^{1}$ Mira Ernkvist, ${ }^{1}$ Lwaki Ebarasi, ${ }^{2}$ Lars Jakobsson, ${ }^{3}$ Arindam Majumdar, ${ }^{2}$ Chunling Yi $^{4}{ }^{4}$ \\ Olivier Birot, ${ }^{1}$ Yue Ming, ${ }^{5}$ Anders Kvanta, ${ }^{5}$ Dan Edholm, ${ }^{3}$ Pontus Aspenström, ${ }^{6}$ Joseph Kissil, ${ }^{4}$ \\ Lena Claesson-Welsh, ${ }^{3}$ Akihiko Shimono, ${ }^{7}$ and Lars Holmgren ${ }^{1,8}$

\begin{abstract}
${ }^{1}$ Department of Oncology-Pathology, Cancer Center Karolinska, Karolinska Institutet, SE-17176 Stockholm, Sweden; ${ }^{2}$ Division of Matrix Biology, Department of Medical Biochemistry and Biophysics, Karolinska Institutet, SE-17177 Stockholm, Sweden; ${ }^{3}$ Department of Genetics and Pathology, Rudbeck Laboratory, Uppsala University, Dag Hammarskjölds väg 20, SE-751 85 Uppsala, Sweden; ${ }^{4}$ Molecular and Cellular Oncogenesis Program, The Wistar Institute, Philadelphia, Pennsylvania 19104, USA; ${ }^{5}$ Department of Clinical Neuroscience, Section of Ophthalmology and Vision, Karolinska Institutet, St Erik's Hospital, SE-11284 Stockholm, Sweden; ${ }^{6}$ Ludwig Institute for Cancer Research, Biomedical Centre, SE-75124 Uppsala, Sweden; ${ }^{7}$ Vertebrate Body Plan, Center for Developmental Biology, RIKEN Kobe, Chuou-ku,
\end{abstract} \\ Kobe 650-0047, Japan
}

The development of the embryonic vascular system into a highly ordered network requires precise control over the migration and branching of endothelial cells (ECs). We have previously identified angiomotin (Amot) as a receptor for the angiogenesis inhibitor angiostatin. Furthermore, DNA vaccination targeting Amot inhibits angiogenesis and tumor growth. However, little is known regarding the role of Amot in physiological angiogenesis. We therefore investigated the role of Amot in embryonic neovascularization during zebrafish and mouse embryogenesis. Here we report that knockdown of Amot in zebrafish reduced the number of filopodia of endothelial tip cells and severely impaired the migration of intersegmental vessels. We further show that 75\% of Amot knockout mice die between embryonic day 11 (E11) and E11.5 and exhibit severe vascular insufficiency in the intersomitic region as well as dilated vessels in the brain. Furthermore, using ECs differentiated from embryonic stem (ES) cells, we demonstrate that Amot-deficient cells have intact response to vascular endothelial growth factor (VEGF) in regard to differentiation and proliferation. However, the chemotactic response to VEGF was abolished in Amot-deficient cells. We provide evidence that Amot is important for endothelial polarization during migration and that Amot controls Rac1 activity in endothelial and epithelial cells. Our data demonstrate a critical role for Amot during vascular patterning and endothelial polarization.

[Keywords: Angiostatin; neovascularization; embryogenesis; GTPase; chemotaxis; transgenic; zebrafish]

Supplemental material is available at http://www.genesdev.org.

Received March 7, 2007; revised version accepted June 22, 2007.

The vascular structure of the developing embryo is formed through two consecutive processes; vasculogenesis, which is the formation of a primitive vascular plexus, and angiogenesis, which is the remodeling and maturation of the initial vascular structure (Risau 1997). Endothelial cell (EC) migration, proliferation, and polarization play fundamental roles in establishing a functional blood vessel network. The development of this network is tightly controlled by growth factors and their receptors, as well as integrins and their extracellular matrix (ECM) ligands (Yancopoulos et al. 1998; Hynes 2002). Vascular endothelial growth factor (VEGF) is a major regulator of embryonic vascular development (Carmeliet et al. 1996; Ferrara et al. 1996) and exerts its

${ }^{8}$ Corresponding author.

E-MAIL lars.holmgren@ki.se; FAX 46-8-339031.

Article is online at http://www.genesdev.org/cgi/doi/10.1101/gad.432007. function by binding to two high-affinity receptor tyrosine kinases, VEGF receptor 1 (VEGFR1) and VEGFR2 (for review, see Olsson et al. 2006), and the coreceptors neuropilin 1 (NP-1) and NP-2 (Soker et al. 1998; Gluzman-Poltorak et al. 2000).

The development of the embryonic vascular system into a highly ordered network requires strict control over the sprouting ECs that are involved in the migration and branching of the early vasculature (Jones et al. 2006). The cells at the tip of the sprout sense the environment, whereas the stalk cells proliferate and form a lumen. Whether cells migrate or proliferate is controlled by VEGF, where a gradient of VEGF induces migration of the tip cells and the concentration of VEGF regulates proliferation of the stalk cells (Gerhardt et al. 2003).

We have shown previously that the membraneassociated protein angiomotin (Amot) is involved in the control of cell migration (Troyanovsky et al. 2001; 
Levchenko et al. 2003). Furthermore, it binds to and mediates the anti-migratory properties of angiostatin through an angiostatin-binding domain (Troyanovsky et al. 2001; Bratt et al. 2005). Amot belongs to a protein family with two additional members-Angiomotinlike-1 (AMOTL-1) and AMOTL-2 - that is characterized by a glutamine-rich domain, a conserved coil-coil domain, and a C-terminal PDZ-binding domain (Bratt et al. 2002). Amot is expressed in two different isoforms with distinct functions: p80-Amot enhances cell migration and stabilizes tubes in vitro, whereas p130-Amot associates with actin and affects cell shape (Troyanovsky et al. 2001; Levchenko et al. 2004; Ernkvist et al. 2006). The functional importance of the PDZ-binding domain is indicated by the migratory defect displayed by cells expressing a C-terminal mutant form of Amot (Levchenko et al. 2003). Transgenic mice expressing the mutant Amot under the EC-specific Tie promoter lose their response to growth factors, which leads to insufficient vascularization and death around embryonic day 9.5 (E9.5) (Levchenko et al. 2003). In addition to the important function during cell migration, Wells et al. (2006) have recently shown that the PDZ-binding domain of Amot functions as a link between Cdc42 activity and the Pals1, Patj, and Par-3 polarity protein complex in epithelial cells.

In this study, we have analyzed the importance of Amot during embryonic angiogenesis, and for this purpose, we studied Amot-deficient mice and Amot knockdown in zebrafish embryos. Amot deficiency leads to vascular defects, including dilated brain vessels and a defective intersomitic vasculature. Our in vitro data demonstrate an important role for Amot during tube formation, with defects in EC polarization and growth factor-induced migration.

\section{Results}

Vascular defects in Amot knockdown zebrafish embryo

The primary vasculogenic and angiogenic vessels in mammals are also present in zebrafish, and the zebrafish embryo has emerged as a useful model to study vertebrate cardiovascular development and physiology (Weinstein 2002; Goishi and Klagsbrun 2004). Thus, to elucidate the role of Amot during embryonic angiogenesis, we used morpholino-mediated knockdown of amot in zebrafish $\left(A m o t^{\mathrm{KD}}\right)$. The zebrafish amot ortho$\log$ was identified by a BLAST search on Ensembl, and two antisense morpholinos were synthesized that were designed to block amot mRNA splicing at exons 2 and 3, respectively. The efficacy of the morpholinos was confirmed by RT-PCR (data not shown), and identical results were obtained using both antisense morpholinos.

To specifically visualize the developing blood vessels, we injected the morpholinos into transgenic zebrafish embryos with the enhanced green fluorescent pro- tein (EGFP) under the endothelial promoter fli-1, Tg (fli1:EGFP) ${ }^{\mathrm{y} 1}$ (Lawson and Weinstein 2002; Isogai et al. 2003). Macroscopic inspection of Amot $^{\mathrm{KD}}$ revealed that they appeared grossly normal during the time of observation (up to $60 \mathrm{~h}$ post-fertilization [hpf]). More detailed analysis using fluorescence microscopy at $36 \mathrm{hpf}$ showed that in $\sim 65 \%$ of the embryos injected with amot antisense morpholinos, the primordial midbrain channel (pMBC) and primordial hindbrain channel (pHBC) were dilated, whereas the other cranial vessels appeared normal (Fig. 1A,B,E; data not shown). This defect was transient, and by $60 \mathrm{hpf}$, both vessels in the $\mathrm{Amot}^{\mathrm{KD}} \mathrm{em}$ bryos were indistinguishable from embryos injected with the mismatch control morpholino (Fig. 1E; data not shown).

$A m o t^{\mathrm{KD}}$ embryos showed severe defects in the formation of trunk vessels. During zebrafish development, the intersegmental vessels (ISVs) originate bilaterally from the dorsal aorta (DA) and the posterior cardinal vein (PCV) beginning around $20 \mathrm{hpf}$, and are thought to form by a process of angiogenesis without cell division (Childs et al. 2002; Isogai et al. 2003). ISV formation is marked by a stereotyped pattern of sprouting and dorsal migration of individual ECs at each somite boundary. Migrating ECs show extensive filopodial extension and retraction as they follow a path initially along the somite boundary and later between notochord and somitic tissue (Leslie et al. 2007). In the control embryos, each ISV-forming cell reached the dorsolateral roof of the neural tube and anastomosed with neighboring ISVs to form the dorsal longitudinal anastomotic vessel (DLAV) (Fig. 1C). In contrast, the sprouting of the ISVs in the $A m o t^{\mathrm{KD}}$ embryos halted midway, resulting in truncated ISVs that failed to form the DLAV (Fig. 1D). Truncated ISVs displayed a "hammerhead" appearance and showed a fivefold reduction in number of filopodia per cell (Fig. 1F). The ISV defect remained at $60 \mathrm{hpf}$, and identical phenotypes were observed using both amot morpholinos.

Thus, morpholino-mediated knockdown in zebrafish revealed that Amot function is required for correct vessel formation and EC migration.

\section{Delayed rescue of the Amot ${ }^{K D}$ phenotype by $A M O T L-1$}

To demonstrate the specificity of the observed defect, we coinjected $100 \mathrm{pg}$ of human amot mRNA with the antisense morpholino, leading to a transient expression of hAmot. This resulted in an almost complete rescue, demonstrating that the vascular defects observed in $\mathrm{Amot}^{\mathrm{KD}}$ are due to loss of amot function (Fig. 1E).

Amot binds to and colocalizes with its family member AMOTL-1 (Supplementary Fig. 1; M. Ernkvist, S. Audebert, P. Lecine, N. Luna-Persson, I. Sinha, M. Liu, A. Bratt, A. Horowitz, K. Aase, and L. Holmgren, in prep.); to assess overlapping functions and potential rescue, murine amotl-1 mRNA was coinjected with the antisense morpholinos, and phenotypes of the embryos were analyzed at 36 and $60 \mathrm{hpf}$. At $36 \mathrm{hpf}$, we observed no rescue 

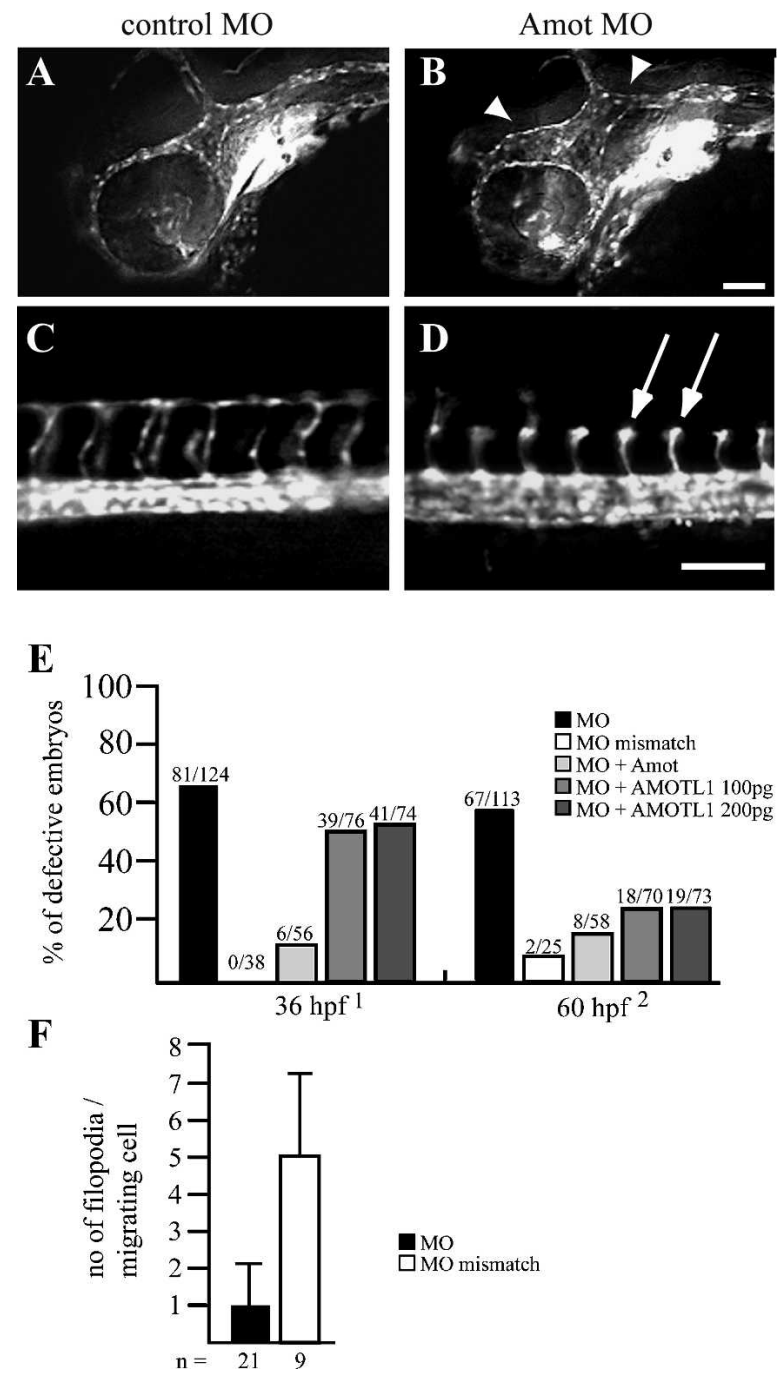

Figure 1. Amot is essential for ISV formation. Depletion of Amot expression in developing zebrafish embryos using amot antisense morpholinos leads to vascular defects in the head and trunk regions. $(A-D)$ Lateral views; dorsal is up, anterior is to the left. The cranial arteries are normal in embryos injected with the mismatch control morpholino $(A)$, whereas the pMBC and $\mathrm{pHBC}$ are dilated in the $A m o t^{\mathrm{KD}}$ embryos ( $B$, arrowheads) at $36 \mathrm{hpf}$. The sprouting of the ISVs and the formation of the DLAV is complete at $36 \mathrm{hpf}$ in the control $(C)$, but the migration of the ECs in the ISVs are halted midway in the $A m o t^{\mathrm{KD}}(D$, arrows), and no continuous SLAV is formed. (E) The percentage of defected embryos at 36 and $60 \mathrm{hpf}$ using different morpholinos. Injection of amot antisense morpholinos leads to $65 \%$ defective embryos at 36 and $60 \mathrm{hpf}$, whereas the control mismatch embryos do not display any defects. Coinjection of human amot mRNA leads to rescue of the phenotypes. Coinjection of murine amotl-1 mRNA leads to a delayed rescue at $60 \mathrm{hpf} .(F)$ The number of filopodia per cell was reduced fivefold in the $A m o t^{\mathrm{KD}}$. $A m o t^{\mathrm{KD}}$ embryos display dilated $\mathrm{pMBC}$ and $\mathrm{pHBC}$, and arrested ISV migration at $36 \mathrm{hpf}$. At $60 \mathrm{hpf}, A m o t^{\mathrm{KD}} \mathrm{em}-$ bryos display only the ISV defects. Bar, $100 \mu \mathrm{m}$.

of the defects, but at $60 \mathrm{hpf}$, there was a partial rescue in which only $26 \%$ of the amotl-1 rescued embryos displayed the ISV phenotype (Fig. 1E).
This result suggests functional redundancy between the two family members but also implies a qualitative difference between Amot and AMOTL-1 function.

\section{Amot is expressed in embryonic blood vessels}

To extend these findings, we further investigated the function of Amot in mice. First we analyzed the amot expression pattern during embryogenesis. Whole-mount in situ hybridization analysis (Supplementary Fig. 1A-D) using amot-specific probes showed that amot was expressed in the intersomitic vessels as early as E8.5 (Supplementary Fig. 1D). Positive signal was also observed in part of the midbrain, the epithelium of the branchial arches, and the limb buds (Supplementary Fig. $1 \mathrm{~A}, \mathrm{C})$. No expression of amot was detected in the aorta after E8.5 or in the heart.

Immunofluorescent staining using anti-Amot-specific antibodies confirmed protein expression in blood vessels in the somitic region (Supplementary Fig. 1H) and the epithelium of the branchial arches (Supplementary Fig. 2C,D). We also detected expression of Amot in capillaries in the brain and neural tube (Fig. 2A; data not shown). Interestingly, Amot was not expressed in all capillaries (data not shown), indicating a spatio-temporal regulation of expression. Furthermore, Amot was not detected in the larger vessels, such as the cardinal vein, aorta, or the heart (Supplementary Fig. 1E; data not shown). Positive staining for Amot was found in the fetal blood vessels (Supplementary Fig. 1Q) and giant cytotrophoblasts of the placenta (Supplementary Fig. 2G). Amot was also expressed in the epithelial cells forming Rathke's pouch (Supplementary Fig. 2A,B), which will later form the anterior pituitary.

\section{Amot-deficient mice die in utero}

We have shown previously that the disruption of the amot gene in mice $\left(\mathrm{Amot}^{-}\right)$results in complete elimination of Amot expression, but also that the penetrance of the amot mutant phenotype is sensitive to genetic background (Shimono and Behringer 2003). Crosses of mice with a $129 / \mathrm{SvEv}$ background lead to morphological defects and death of $\sim 70 \%$ of the Amot-deficient embryos around E7.5. However, backcrossing once into the C57/ B6 (129B6 mixed) background resulted in 50\% dead embryos at the same time point (E7.5) (Shimono and Behringer 2003). In this study, we backcrossed the mice further into the C57/B6 background (up to six backcrosses), and in contrast to previous findings, we found that Amot-deficient embryos survived at a near Mendelian ratio after E7.5 (E8.5-E10.5) (Supplementary Table 1; Supplementary Fig. 3). Nevertheless, the number of live Amot-deficient pups was only $\sim 25 \%$ of the expected ratio, indicating that a majority die in utero (Supplementary Table 2; Supplementary Fig. 3). To study when the Amot ${ }^{-}$embryos died, we established timed matings and analyzed embryos between E11.5 and E17.5. We detected similar ratios of survival in these embryos as we did in the $A_{m o t^{-}}$pups (Supplementary Table 3; Supplementary 
Fig. 3). To further pinpoint when they died, we analyzed them at E11 and found that the frequency were close to a Mendelian ratio (Supplementary Table 4). This supports the argument that the embryos survive past E7.5 in a C57/B6 background, but that a majority of the Amot $^{-}$embryos die between E11 and E11.5 (Supplementary Fig. 3).

\section{Amot-negative yolk sacs display vascular defects}

A primitive vascular network in the yolk sac is first established through vasculogenesis, followed by the development of a more mature network through angiogenesis around E8-E8.5. Angiogenesis in the embryo proper takes place around the same time, but blood cells are not present in the vessels until E8.5, when the embryonic and yolk sac vascular systems amalgamate and the nucleated red blood cells formed in the yolk sac enter the embryo. We analyzed the vascular network of the yolk sacs by immunofluorescent staining of the ECs using an antibody against the endothelial marker PECAM (platelet/endothelial cell adhesion molecule). The yolk sacs from wild type or heterozygote embryos at E10.5 exhibited branched structures and a highly organized vascular bed (Fig. 2D,F), whereas $A_{m o t}^{-}$yolk sacs displayed an abnormal vascular patterning (Fig. 2E,G). The Amot yolk sacs contained vessels with increased diameter, and some vessels anastomosed to form lagoon-like structures (Fig. 2G). Erythropoiesis was not dependent on Amot, as we could observe nucleated blood cells in the Amot yolk sacs. A functional blood flow between the yolk sac and the embryo proper was also established, as we detected red blood cells in the embryo before embryonic erythropoiesis at E11.

Figure 2. Vascular defects in the Amot-deficient yolk sacs and embryos at E10.5. Immunofluorescent staining at E10.5 using Amot-specific antibodies in combination with the endothelial marker isolectin-B4. Amot is expressed by the capillaries of the brain $(A)$ where the staining overlaps with isolectin, a marker for EC $(A-C)$. More expression data are found in Supplementary Figures 1 and 2. Wild-type yolk sacs form a branched vascular network with different sized vessels $(D)$, whereas the Amotdeficient yolk sacs display a less-organized vessel network $(E)$. At a higher magnification, the difference is shown more clearly, with narrow blood vessels in the wild-type $(F)$ and dilated blood vessels that have anastomosed into lagoon-like structures (asterisk) in the $A m^{-}{ }^{-}$yolk sacs $(G)$. Stage-matched E10.5 embryos of wild type $(H)$ and $\operatorname{Amot}^{-}(I)$. (J) PECAM staining of the blood vessels in the brain show that in wild-type embryos, the blood vessels have expanded into a vascular plexus with large vessels that branch into smaller capillaries in the developing brain and somites (arrows). (K) The brains of the $A m o t^{-}$embryos show similar structures as in the yolk sacs with dilated vessels (arrows) and lagoon-like structures (asterisk). There is an accumulation of erythrocytes in the $A \mathrm{mot}^{-}$vessels $(M)$, compared with the wild-type vessels $(L)$. There is a lack of paraneural capillaries in the somitic region in the $A_{m o t}{ }^{-}$embryos $(O$, arrowhead) that are present in the wild-type embryos $(N$, arrowheads). Bars: $A-C, 14 \mu \mathrm{m} ; D, E, H, I, 1 \mathrm{~mm} ; F, G, 21 \mu \mathrm{m} ; J-O$, $10 \mu \mathrm{m}$.
Amot is necessary for normal blood vessel formation

We found that at E10.5, the Amot ${ }^{-}$embryos and placentas were $85 \%$ and $75 \%$ the size of their littermates, re-
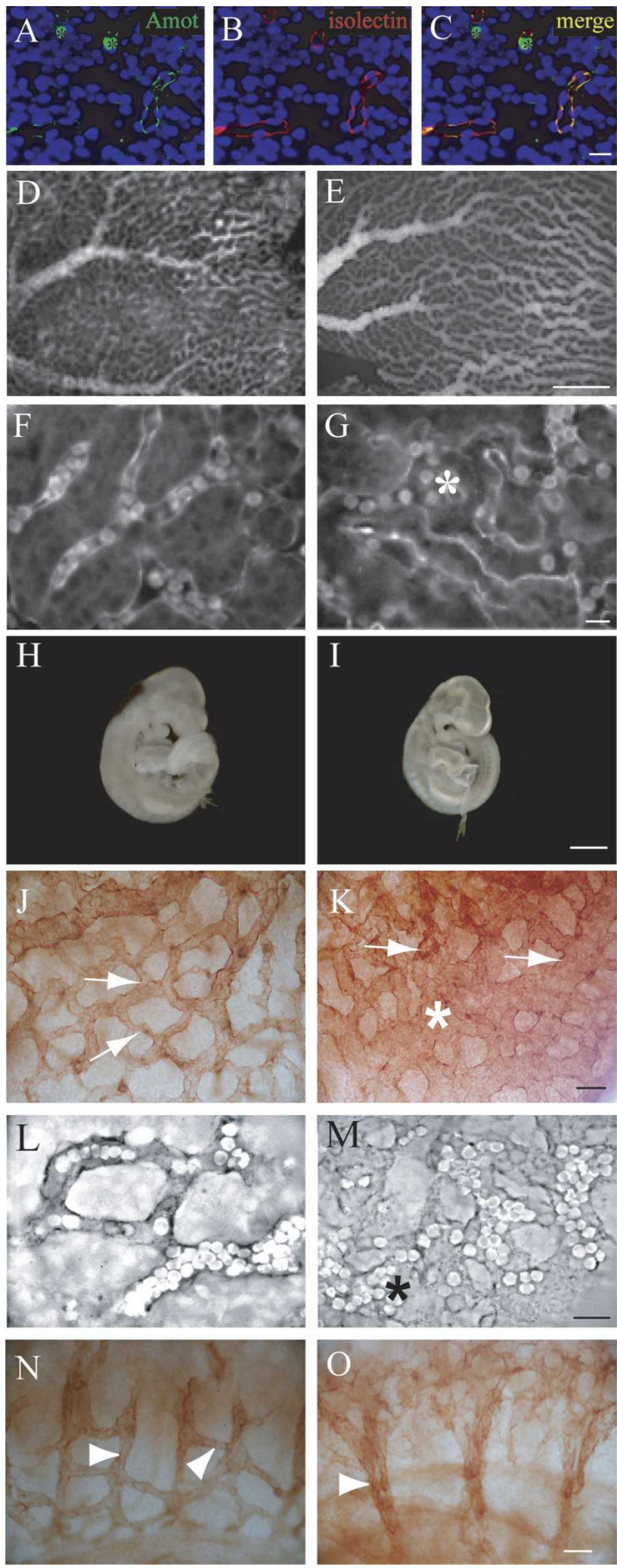
A

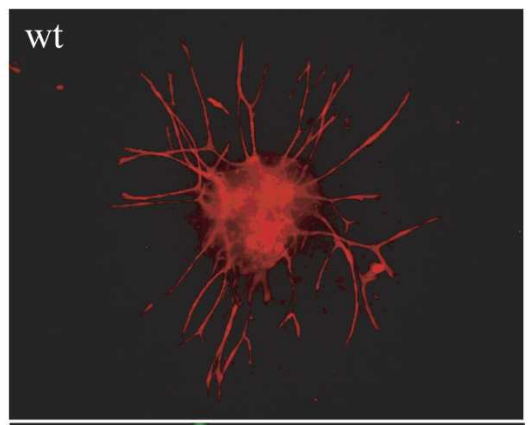

C

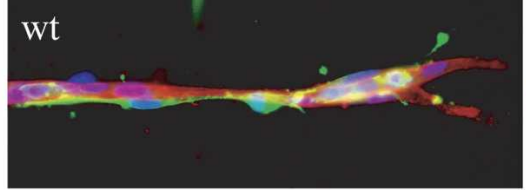

D

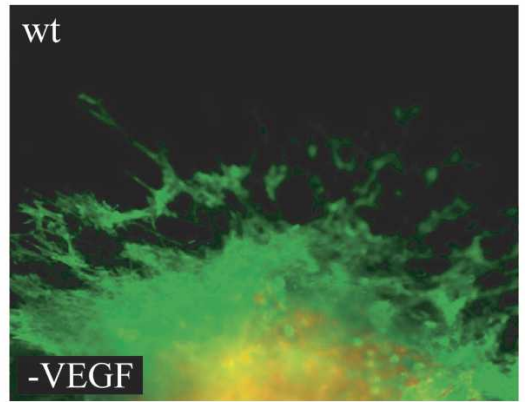

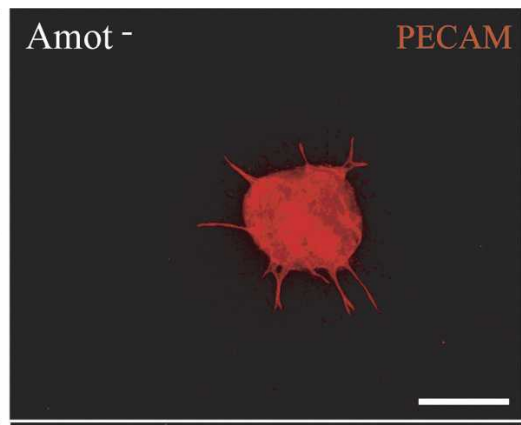
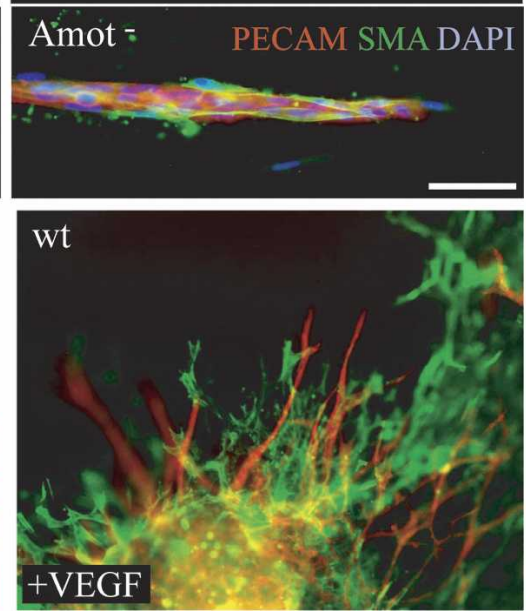

B
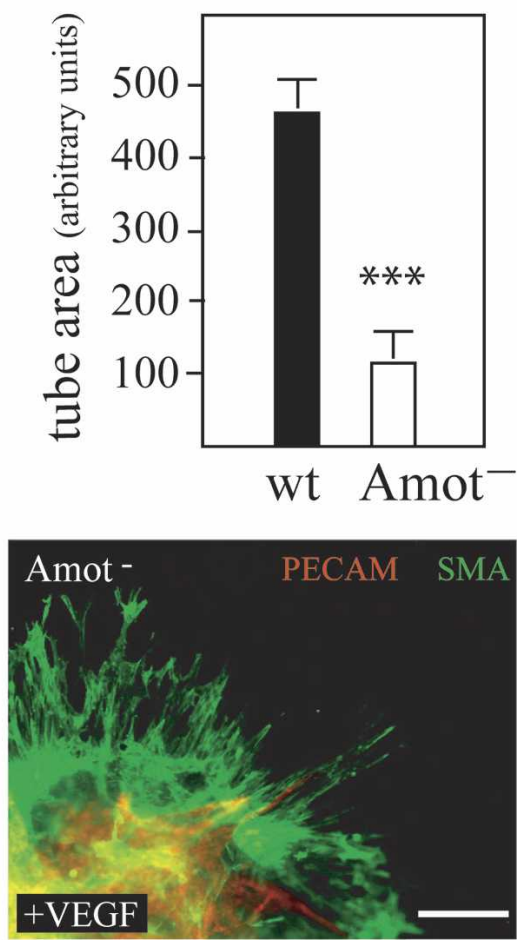

Figure 3. VEGF-induced sprouting is impaired in Amot-deficient embryoid bodies. ES cells were allowed to differentiate and grow in three-dimensional collagen I gels. ECs were visualized with the PECAM antibody (red) and supporting perivascular cells with $\alpha$-smooth muscle actin ( $\alpha$ SMA) (green). (A) In the presence of VEGF, the wild-type cells migrate and form sprouting tubes that invade the collagen gel, whereas in the Amot ${ }^{-}$cells only a few sprouts are formed. (B) Quantification of the tubes that invade the collagen I gel. $(C)$ Invading tubes were coated with perivascular cells in both the wild-type and Amot $^{-}$bodies. $(D) \alpha$ SMA-positive cells were not dependent on either VEGF or Amot for their migration. Bars: $A, 500 \mu \mathrm{m} ; B, 50 \mu \mathrm{m} ; C, 250 \mu \mathrm{m}$.

spectively (Supplementary Fig. 4A,B,D), indicating that the growth of both embryonic and extraembryonic tissues was affected by the Amot deficiency. Immunofluorescence analysis showed that Amot was expressed in both blood vessels and cytotrophoblasts of the placenta (Supplementary Figs. 1Q, 2G), and we examined the possibility that the observed growth retardation was due to placental deficiency. Histological analysis of the wildtype and $A m o t^{-}$placentas did not reveal any obvious defects; thus we conclude that the establishment of the placental layers and function was normal in the Amotdeficient placentas (Supplementary Fig. 4C).

At E10.5, the wild-type embryo has developed an intricate system with vessels supporting the developing brain and somites. Amot is expressed in the capillaries at this time point, and we decided to focus our analysis on the cephalic and somitic regions. The blood vessels of the embryos were visualized by whole-mount staining of the ECs using the PECAM antibody. The vessel network in the wild-type brain was well developed and consisted of larger vessels that branched into smaller capillaries (Fig. 2J,L). In contrast, the Amot-deficient embryos displayed a less structured network that formed lagoon-like structures with an accumulation of erythrocytes, comparable with the ones detected in the yolk sac (Fig.
$2 \mathrm{~K}, \mathrm{M})$. The branching was not affected in the $A m t^{-}$ embryos, but the vessel diameter was twice the size compared with wild-type embryos (Supplementary Fig. $5 \mathrm{~B}$; data not shown). The intersomitic blood vessels in the wild-type embryos formed an organized network, whereas the interconnecting paraneural blood vessels were missing in the $\mathrm{Amot}^{-}$embryos, and the remaining vessels appeared dilated and shorter (Fig. 2N,O). A similar difference in vessel morphology in the brain and the somitic region was also observed at E9.5 and E11 (Supplementary Fig. 5A; data not shown).

Amot was also expressed by a subset of epithelial cells, but we did not observe any obvious defects in the giant cytotrophoblasts of the placenta (Supplementary Fig. 2K) or in the patterning of the branchial arches or limb buds (Fig. 2I), suggesting that Amot is not essential for establishing these epithelial structures.

The defects observed both in the embryos and yolk sacs in $A$ mot $^{-}$embryos show that Amot is dispensable for vasculogenesis, but plays an important role in angiogenesis.

VEGF-induced tubulogenesis is impaired in embryoid bodies lacking Amot

To investigate the role of Amot in tubulogenesis, we assessed the tube-forming potential of wild-type and 
Amot ${ }^{-}$embryonic stem (ES) cells. To this end, we used the embryoid body assay, in which ES cells are allowed to differentiate and form a primitive vascular plexus (Vittet et al. 1996). This assay mimics vasculogenesis and angiogenesis and is widely used to investigate the different steps involved. Amot is located on the X chromosome, and the ES cells used were male, thus providing us with an Amot-deficient cell after only one recombination event. Wild-type embryoid bodies expressed both isoforms of Amot, both by ECs and by epithelial-like cells (Supplementary Fig. 6A,D-G), whereas the Amot $^{-}$ embryoid bodies lacked Amot (Supplementary Fig. 6A).

We used a model for invasive angiogenesis to analyze the developing vascular structures in the wild-type and Amot ${ }^{-}$embryoid bodies. The embryoid bodies were cultivated in three-dimensional collagen I gels and stimulated with VEGF to induce outgrowth of tubes. In agreement with our findings from the $A m t^{-}$embryos, we observed no defect in the ability of the $A \mathrm{mot}^{-}$ES cells to differentiate into ECs (Fig. 3).

In the presence of VEGF, the wild-type ECs invaded and extended tubes covered with perivascular smooth muscle cells into the collagen matrix (as described previously) (Fig. 3; Jakobsson et al. 2006). In contrast, the Amot $^{-}$ECs did not respond to VEGF, and only one-fifth of the amount of PECAM-positive tubes was observed invading the matrix (Fig. 3). Smooth muscle cells invaded the matrix in the absence of VEGF and were not affected in the Amot $^{-}$embryoid bodies (Fig. 3C,D), showing the EC-specific effect of Amot deficiency.

To exclude the possibility that the observed difference was due to a deficit of ECs in the Amot ${ }^{-}$embryoid bodies, we measured the expression of five different endothelial markers (PECAM, VEGFR1, VEGFR2, VE-cadherin, and NP1) using real-time PCR. There was no significant difference in the expression levels of the EC-specific markers between wild-type and $\mathrm{Amot}^{-}$embryoid bodies (Supplementary Fig. 6B). Neither was the proliferative response of the ECs toward VEGF affected (Supplementary Fig. 6C).

The difference between the in vivo situation and the severe phenotype observed in the embryoid bodies with major defects in migration and tube-forming potential of the ECs suggests that there is a developmental pressure that compensates the lack of Amot, leading to a less severe phenotype in vivo. To test the influence of Amot on the angiogenic process in vivo without the possibility for developmental up-regulation of compensatory signaling pathways, we used the choroidal neovascularization (CNV) model, in which choroid vessels in the eye are stimulated to grow into the subretinal space. This process is driven by VEGF and shares similar features with the angiogenic sprouting observed in the embryoid body system (Kvanta 2006). The CNVs were generated by krypton laser-induced rupture of the Bruch's membrane (the innermost layer of the choroid), as described in Materials and Methods. Control and Amot small inhibitory RNAs (siRNAs) were injected intraocularly at 0,3 , and $6 \mathrm{~d}$ post-treatment. We verified the efficacy of Amot siRNA knockdown in mouse ECs (Supplementary Fig. 6H). The angiogenic response was calculated using the PECAM area of the plaque. As shown in Figure 4, the angiogenic response in the Amot siRNA-treated CNVs was half that of the control siRNAtreated CNVs. Taken together, the embryoid body and $\mathrm{CNV}$ results indicate that there is a pressure to control the expression of angiogenic-related genes during mouse development.

Coinjection of amotl-1 mRNA with the antisense amot morpholinos can rescue the phenotype in $\mathrm{Amot}^{\mathrm{KD}}$ zebrafish embryos (Fig. 1E) and may potentially rescue the phenotype in $\mathrm{Amot}^{-}$mice as well. We investigated the expression pattern of AMOTL-1 and AMOTL-2 during mouse development, and AMOTL-1, but not AMOTL-2, overlaps with that of Amot (Supplementary Figs. 1H-P, 2E-L). It is therefore conceivable that AMOTL-1 is one of the genes that rescue the effect of Amot deficiency in the mouse.

These studies demonstrate that Amot does not influence the differentiation or proliferation of ECs but is important for proper migrational and tube-forming response to growth factors, such as VEGF.
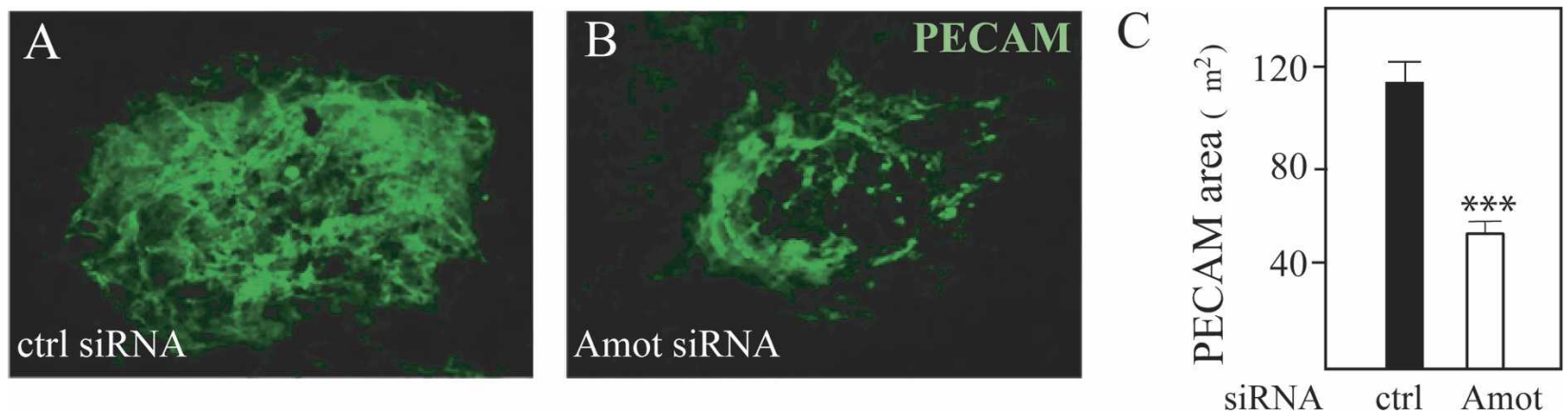

Figure 4. siRNA knockdown of Amot in the CNV model impairs angiogenic sprouting in vivo. Neovascularization was induced by laser-induced rupture of the innermost layer of the choroid. The lesions were treated with intraocular injections of siRNA at 0,3 , and $6 \mathrm{~d}$. The sprouting ECs within the lesion were visualized using the PECAM antibody. Mice treated with the control siRNA $(A)$ display extensive sprouting in the lesion, whereas the sprouting area of the lesion in mice treated with the Amot siRNA $(B)$ is half the size $(C) .\left(^{\star \star \star}\right) P<0.001$. 
Amot is important for proper focal adhesion formation

To investigate the cellular mechanism behind the observed defects, we immortalized ECs from the embryoid bodies by the endothelial-specific polyoma middle $\mathrm{T}$ $(\mathrm{PmT})$ virus as described in Materials and Methods. Amot expression in the immortalized ECs (PmT-ECs) was analyzed by Western blot. As shown in Figure 5A, the wild-type PmT-ECs expressed both the p80 and p130 isoform of Amot, whereas the Amot PmT-ECs did not. We verified that the isolated cells were ECs and expressed equal amount of the endothelial-specific markers by real-time PCR, FACS, and Western blot (Fig. 5B; Supplementary Fig. 7A,B).

We and others have shown that Amot localizes to tight junctions but is not essential for their formation (Bratt et al. 2005; Wells et al. 2006). As expected, both the wildtype and Amot ${ }^{-}$PmT-ECs formed tight junctions as indicated by the tight junction-specific marker zonula occludens-1 (ZO-1), although only the wild-type PmT-ECs express Amot (Fig. 5C).

To analyze whether cells lacking Amot differed in cytoskeletal organization compared with the wild-type cells, we stained subconfluent cells for actin and the focal adhesion marker paxillin. We found a significant difference between wild-type and Amot $^{-}$PmT-ECs with re- spect to actin fiber organization and paxillin staining. Wild-type cells displayed a regular pattern of actin fibers with paxillin staining at the actin fiber ends (Fig. 5D). The Amot ${ }^{-}$cells, on the other hand, revealed a disorganized pattern of actin fibers and smaller and shorter focal adhesions, which were not ordered along the actin fibers (Fig. 5D). To quantify the difference, we measured the area and the length of the focal adhesions. As shown in Figure 5, E and F, Amot-deficient cells contained $20 \%$ shorter and smaller focal adhesions compared with wildtype cells. Similar staining patterns were also obtained using the focal adhesion kinase (FAK) and vinculin-specific antibodies (Supplementary Fig. 7C).

Amot is thus likely to play a role in controlling the structural integrity of ECs by affecting the organization of actin fibers and focal adhesions, thereby affecting cell shape.

\section{Amot is involved in migration}

We evaluated the migratory capacity of the PmT-ECs using two independent migration assays: the Boyden chamber assay in which single cells are allowed to migrate toward a chemoattractant, and the in vitro wound healing assay in which a wound is made in a confluent

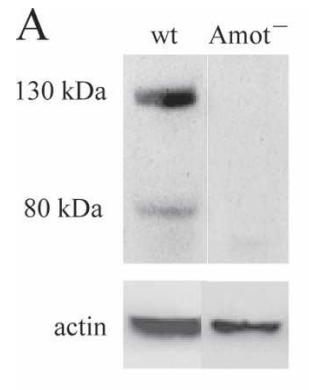

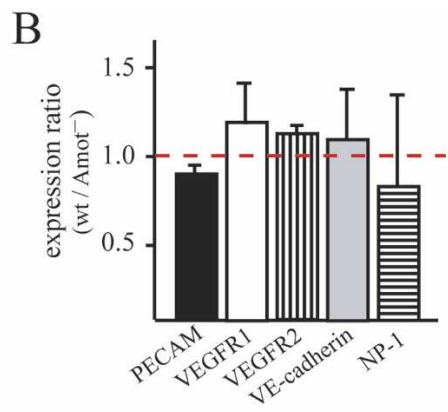
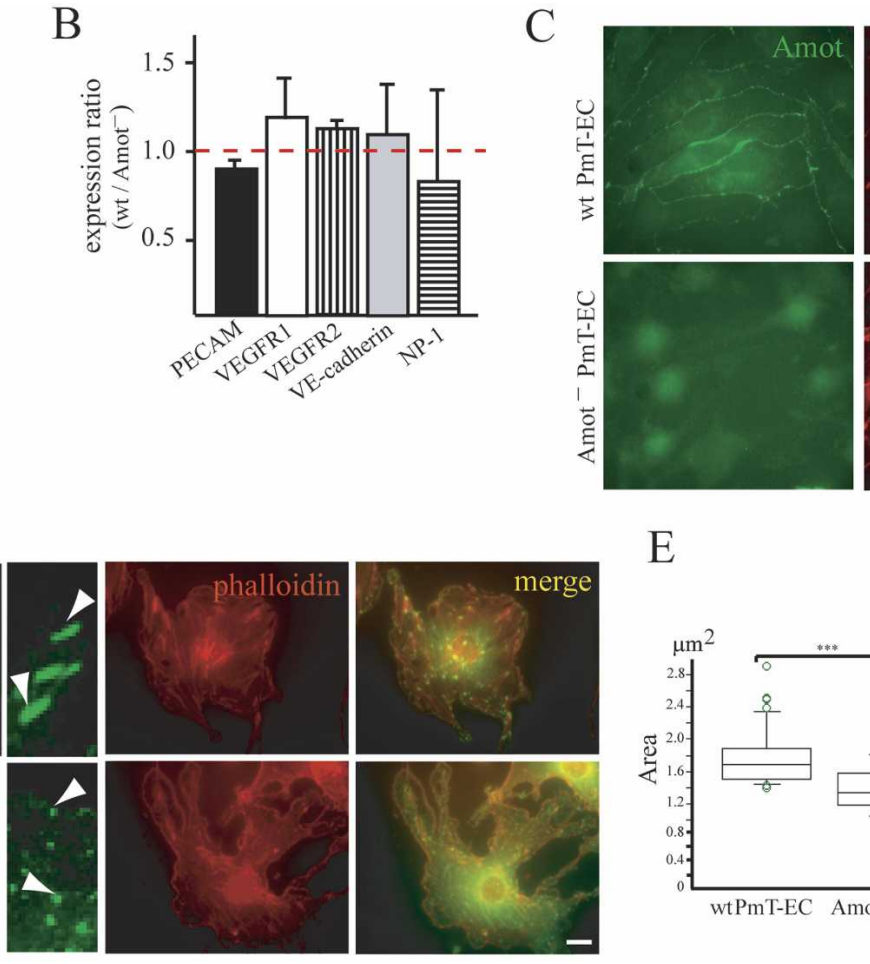

E
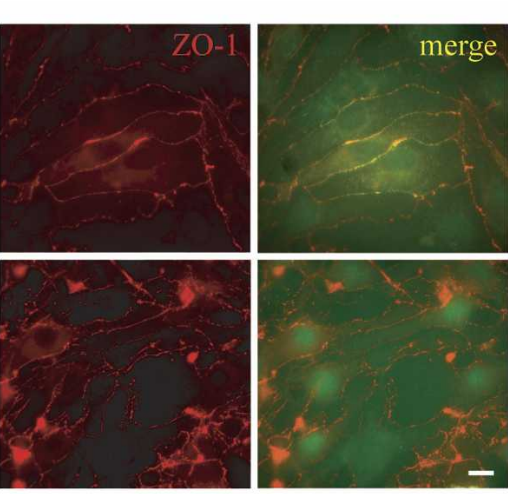

F
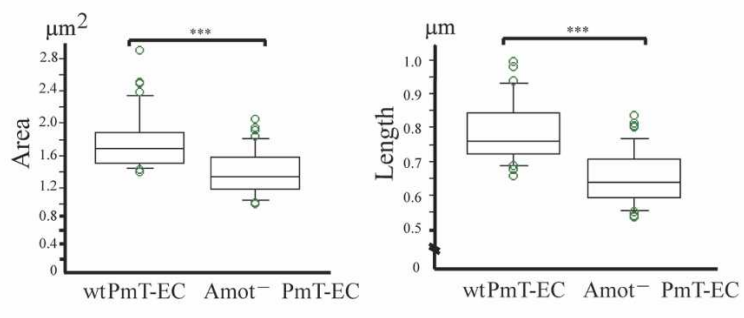

Figure 5. Amot is not required for tight junction formation, but for the organization of actin and focal adhesions. (A) Wild-type PmT-ECs express both isoforms of Amot, whereas no expression is detected in the $A m t^{-}$PmT-ECs. (B) RT-PCR analysis shows that both cell lines express an equal amount of EC-specific markers. (C, top panel) Amot expression overlaps with the tight junction marker ZO-1 in cell-cell contacts in the wild-type PmT-EC. (Bottom panel) ZO-1 still localizes to cell-cell junctions in the absence of Amot. $(D)$ Visualization of focal adhesions (arrowheads) and actin fibers using a paxillin antibody and phalloidin. (Bottom panel) The Amot ${ }^{-}$ PmT-EC displays an irregular pattern of actin fibers and shorter and smaller focal adhesions. The highlighted areas show focal adhesions at a higher magnification. Box diagram showing the area $(E)$ and length $(F)$ of focal adhesions. Bar, $14 \mu \mathrm{m}$. $\left(^{\star \star \star}\right) P<0.001$. 
Aase et al.

cell layer and cells are subsequently allowed to migrate toward the wound. The latter assay measures spontaneous migration of a sheet of cells, and the two assays are known to evoke different responses in the Rac/Rho pathway (Aepfelbacher et al. 1997; Nobes and Hall 1999).

The Amot ${ }^{-}$PmT-ECs displayed a threefold reduction in the basal migrating capacity compared with the wildtype PmT-ECs in the Boyden chamber assay (Fig. 6A). Consistent with the results from the embryoid body assay, the Amot ${ }^{-}$PmT-ECs did not exhibit a chemotactic response to VEGF or to basic fibroblast growth factor (bFGF) (Fig. 6A). Furthermore, the Amot PmT-ECs showed a similar lack of response to VEGF in the wound healing assay (Fig. 6C). The observed difference in growth factor response between the two cell lines was not detected in serum (Fig. 6A,D). To verify our data in another cell system, we performed knockdown of Amot in bovine capillary endothelial (BCE) cells using amotspecific siRNA. Both isoforms of Amot were efficiently knocked down (Fig. 6G), and the result was similar to what we observed in the PmT-ECs, with a decrease in both basal and bFGF-induced migrational rate in the Amot siRNA-transfected cells (Fig. 6H).

Our data demonstrate the importance of Amot for proper migrational response toward growth factors during both single cell migration and sheet-like movement.
Figure 6. Amot is critical for growth factor-mediated migration. (A) Wild-type and $A$ mot $^{-}$PmT-ECs were allowed to migrate toward VEGF, bFGF, or serum in the Boyden chamber assay. Amot PmT-ECs fail to respond to growth factors but show an equal migratory capacity as wild-type PmT-ECs in the presence of serum. In the in vitro wound healing assay, wild-type PmT-ECs respond to VEGF with an increase in migration rate $(B)$, whereas the $A m o t^{-}$PmT-ECs fail to respond $(C)$. $(D)$ There is no difference in migratory response in the presence of serum. $(E, F)$ Both wild-type and $A m o t^{-}$PmT-ECs display a similar response in proliferation to VEGF. (G) siRNA knockdown of Amot in BCE cells was confirmed using Western blot. (H) Amot siRNA-transfected BCE cells show a decrease in basal migration in the Boyden chamber assay and do not respond to bFGF.

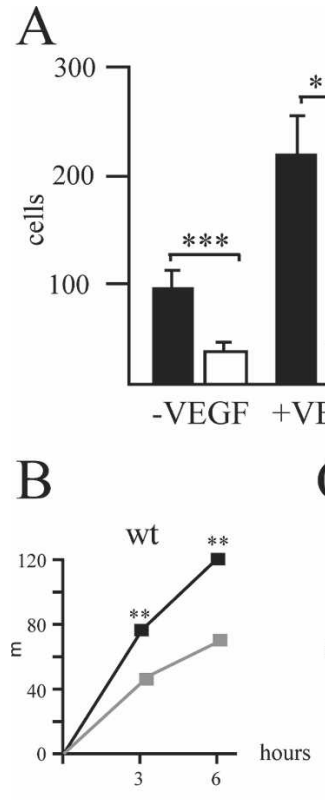

E

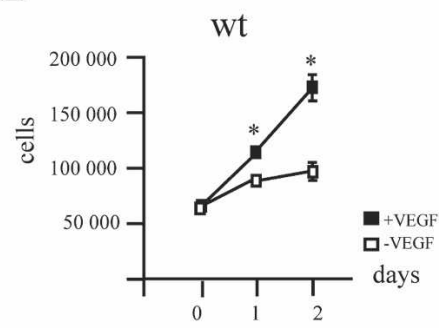

F

$\mathrm{G}$
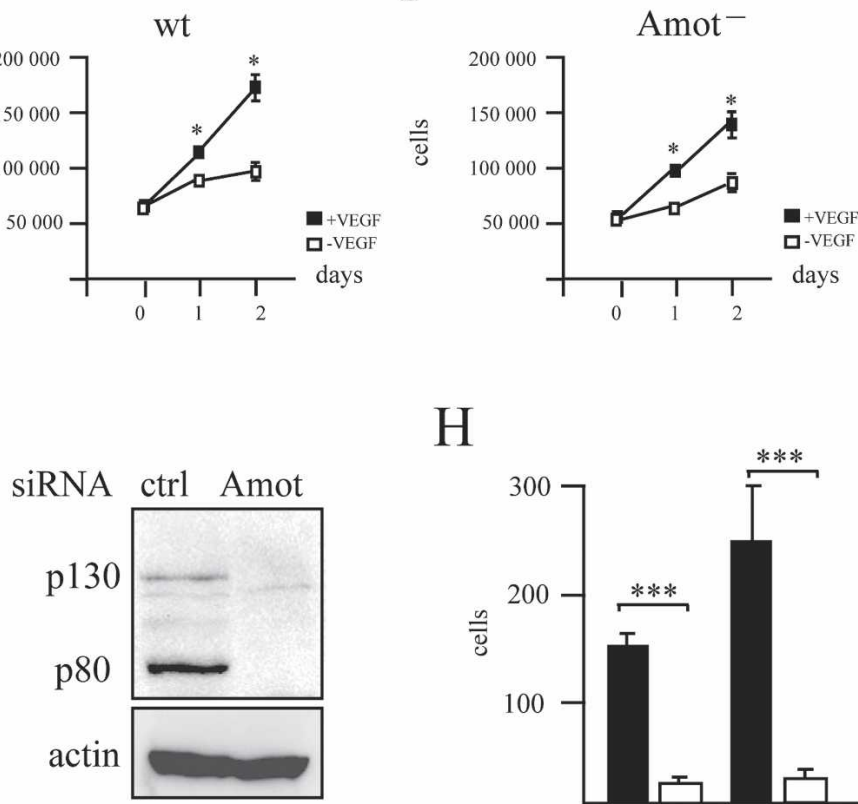

$\mathrm{H}$

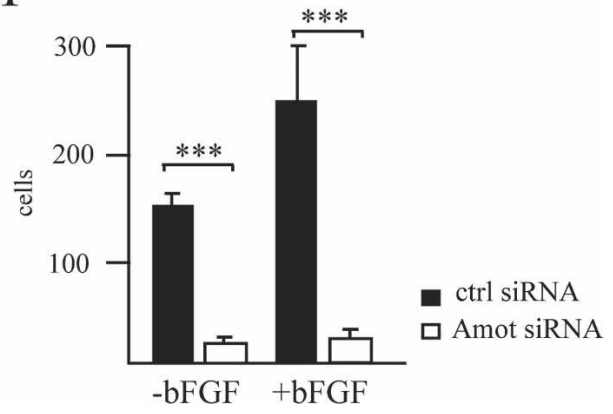


The observed defect was not due to a general defect in the VEGFR2-signaling, since the wild-type and $\mathrm{Amot}^{-}$ cells displayed a similar proliferative response upon VEGF treatment (Fig. 6E,F) and expressed equal levels of VEGFR1, VEGFR2, and NP-1 (Fig. 5B).

We studied the dynamics of sheet-like or single cell migration by time-lapse photography. During single cell migration, the wild-type PmT-ECs developed one large lamellipodia in the direction of migration (Fig. 7A; Supplementary Movie 1). In contrast, the $A_{m o t}^{-}$PmTECs exhibited an unpolarized phenotype, with several peripheral lamellipodia and cell protrusions that expanded and contracted rapidly (Fig. 7A; Supplementary Movie 2). The number of cell protrusions was increased fourfold in the Amot ${ }^{-}$PmT-ECs compared with the wildtype cells (Supplementary Fig. 6D). Since we detected a reduction in migration capacity of the $\mathrm{Amot}^{-}$PmT-ECs in our migrational assays, we tracked the migration of the cells to characterize their migration pattern. We found that the distance the wild-type cells migrated was five times as long as the $\mathrm{Amot}^{-}$cells (Supplementary Fig. $7 \mathrm{E}, \mathrm{F})$, although the expansion and contraction of the protrusions was faster in the Amot $^{-}$cells (Fig. 7A; Supplementary Movies 1, 2).

\section{Amot-deficient cells show a defect in polarization}

Cell polarization and the establishment of functionally specialized domains have been shown to play a pivotal role in the directional movement of cells in a chemotactic gradient (Franca-Koh and Devreotes 2004). The observation that Amot $^{-}$PmT-ECs displayed a defect in migration led us to investigate the ability of these cells to polarize. In a migrating cell, the Golgi apparatus is oriented in the direction of migration, where it is responsible for the polarized delivery of vesicles and their subsequent insertion at the plasma membrane (Singer and Kupfer 1986). The Golgi apparatus is thus used as an indicator for the degree of polarization of a cell, where a cell is defined as polarized if the Golgi apparatus is located within one $120^{\circ}$ sector. We stained subconfluent cells with a Golgi-specific antibody, divided each cell in three sectors, and measured the extent of polarization. Staining of wild-type PmT-ECs revealed that a majority of the cells had their Golgi apparatus located within one sector, whereas only about half of the Amot ${ }^{-}$PmT-ECs showed the same pattern (Fig. 7B,C).

Rac1 activity is increased in Amot-deficient and Amot knockdown cells

Cdc42 is important to maintain cell polarity (Nobes and Hall 1995), and since the Amot ${ }^{-}$PmT-ECs were less polarized compared with wild-type cells, we investigated the levels of activated Cdc42. We were unable to detect any GTP-bound Cdc42 in the PmT-ECs /data not shown), indicating that it is not active or that the levels were too low for detection.
The superfluous protrusions and lamellipodia formed by the Amot ${ }^{-}$PmT-ECs led us to study the levels of activated Rac1, an important modulator of lamellipodia formation. Experiments revealed an increase of activated Racl in the Amot $^{-}$PmT-ECs compared with wildtype PmT-ECs (Fig. 7D), consistent with the observed increase in protrusions and lamellipodia (Fig. 7A; Supplementary Movie 2). Rac activity is controlled by guanine nucleotide exchange factors (GEFs) and GTPaseactivating proteins (GAPs). One such GAP is Rich-1 (RhoGAP interacting with CIP4 homologs), shown to be a GAP for both Rac1 and Cdc42 (Richnau and Aspenstrom 2001; Wells et al. 2006). Recent findings have shown that Amot acts as a scaffolding protein that associates to Rich-1 (Wells et al. 2006), and we were interested in whether the expression of Rich-1 was altered in the $\mathrm{Amot}^{-}$cells. Western blotting using antiRich-1 antibodies showed that there is an equal expression level of Rich-1 in both cell lines (Fig. 7F). The localization of Rich-1 in subconfluent cells revealed that in addition to a cytoplasmic distribution, Rich-1 was also expressed at the edge of lamellipodia in wildtype cells, whereas that pattern was lacking in the Amot $^{-}$cells (Fig. 7G). This indicates that there is a local dysregulation of Racl activity leading to increased lamellipodia formation and a total up-regulation of GTP-Rac1.

As a step in immortalizing ECs, PmT recruits and activates Src family tyrosine kinases, which stimulates signaling to both the MAP kinase and PI3K pathways (Ong et al. 2001). To verify that the observed difference in Racl activation between the wild-type and Amot ${ }^{-}$PmT-ECs was not caused by a synergistic effect from the PmT virus and the lack of Amot, we depleted Amot in 293T cells using siRNA. These cells are of epithelial origin and express both isoforms of Amot endogenously. The Amot knockdown cells showed an increase in Rac1 activity consistent with the $\mathrm{Amot}^{-} \mathrm{PmT}$ EC data (Fig. 7E), indicating that the result from the immortalized ECs was not an effect of PmT-induced subversion of Racl activity. In addition, the experiment shows that the Amot control of Racl activity is not EC specific.

\section{Discussion}

We silenced Amot expression in zebrafish, mouse, and ECs and provided evidence for an important role of Amot in vascular reorganization and EC migration. Amot was primarily expressed by ECs of intersomitic vessels as well as brain capillaries. No detectable mRNA or protein expression could be detected in larger vessels such as the aorta or the cardinal vein. Amot was also detected in epithelial structures of the branchial arches and Rathke's pouch. We have shown previously that Amot is up-regulated in tumor endothelium of both human and mouse tumors but is not detectable in vessels of normal tissues (Troyanovsky et al. 2001; Holmgren et al. 2006). Further- 
Aase et al.

Figure 7. Amot deficiency results in an increase in lamellipodia formation, an increase in Rac activity, and loss of polarization. ( $A$, top panel) Photographs from time-lapse studies at indicated time points show that the wild-type PmT-ECs form one large lamellipodia (asterisk) in the direction of migration. (Bottom panel) In contrast, the Amot $^{-}$PmT-ECs form several cell protrusions and lamellipodia (arrows). (B) Subconfluent cells are stained with a Golgi apparatus-specific antibody and scored for degree of polarization. $(C)$ A majority of the wild-type cells are polarized, whereas only about half of the Amot cells display the same pattern. Lamellipodia are highlighted with white dotted lines in A. Bar, $14 \mu \mathrm{m}$. (D) Racl activation is increased in the $A$ mot $^{-}$ PmT-ECs. (E) Knockdown of both isoforms of Amot in 293T cells also leads to an increase in the activation of Racl. (F) Equal expression of Rich-1 in wild-type and $A_{m o t}{ }^{-}$PmT-ECs. $(G)$ In wild-type cells, Rich-1 localizes to lamellipodia (arrow, top panel), but its localization is altered in the Amot $^{-}$PmT-ECs (bottom panel).
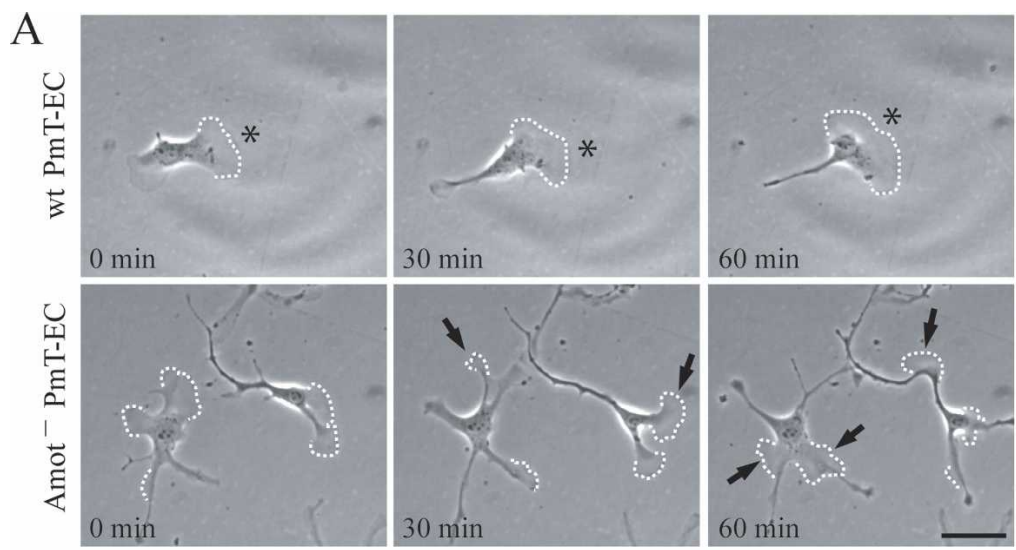

B
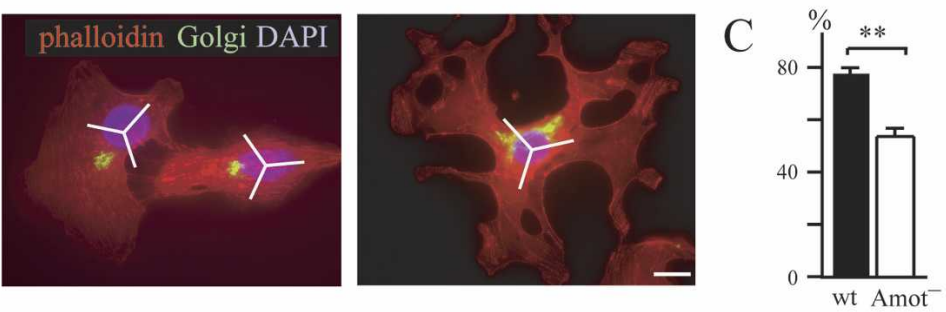

$\mathrm{D}$
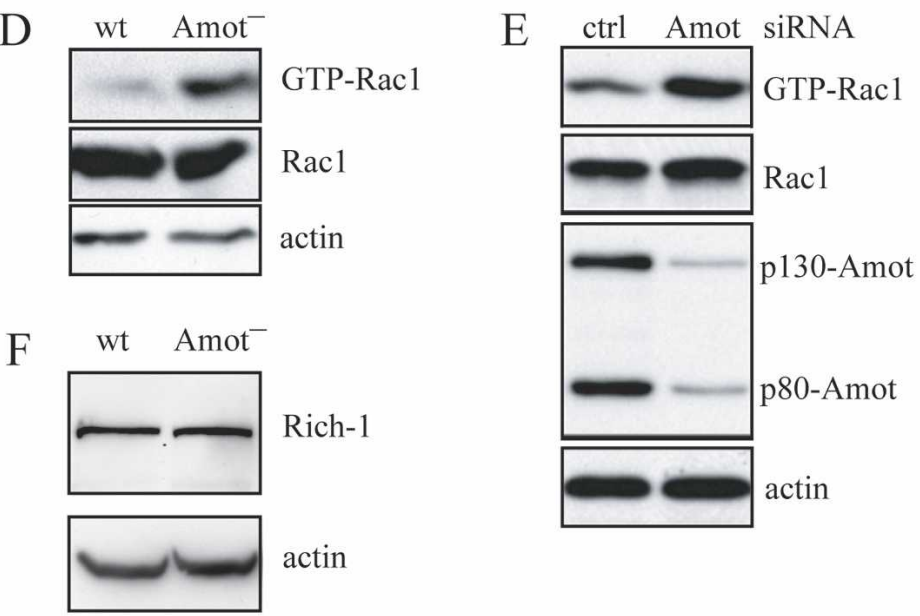

Rich-1
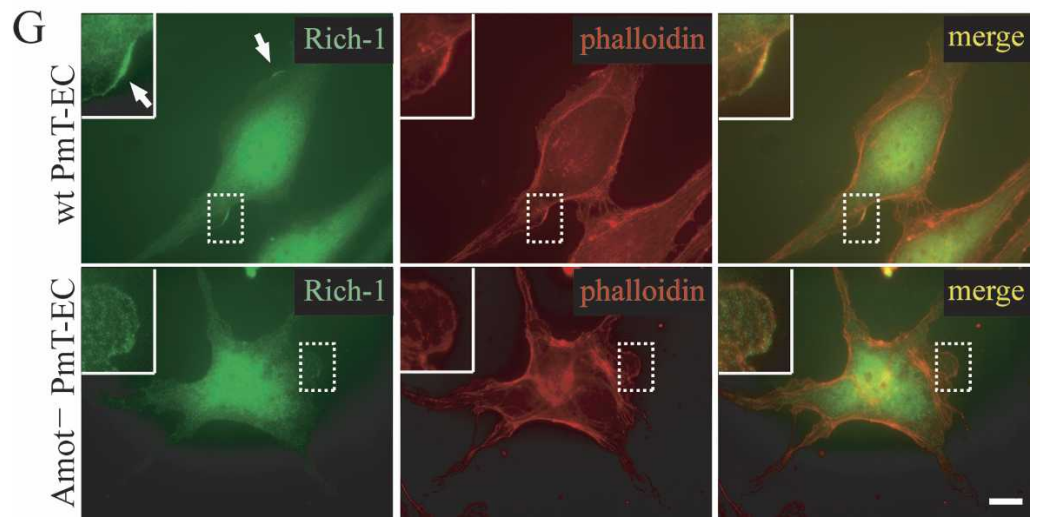

more, Amot is expressed in ECs during normal physiological angiogenesis of the mouse retina (Bratt et al. 2005). Taken together, these findings indicate that Amot is primarily expressed in angiogenic tissues.

\section{Amot in angiogenesis}

We have previously shown that ectopic expression of Amot promotes migration of ECs. Furthermore, angio- 
statin (which binds Amot) or Amot antibodies inhibit EC migration in the Boyden chamber assay (Troyanovsky et al. 2001; Bratt et al. 2005; Holmgren et al. 2006; T. Levchenko, N. Veitonmäki, H. Gerhardt, Y. Ming, A. Kvanta, K. Berggren, R. Carlsson, and L. Holmgren, in prep.). Our in vivo data support the notion that Amot plays a positive role in neovascularization and more specifically EC migration. Knockdown of Amot protein expression in zebrafish resulted in a distinct migratory defect of the ISVs. Capillary sprouting from the DA or PCV was not affected, but the vessels failed to migrate and form functional DLAVs. Interestingly, Amot ${ }^{\mathrm{KD}}$ phenocopies the loss of VEGFR2 kinase activity in zebrafish (Covassin et al. 2006). The partial formation of arteries by the VEGFR mutant was most likely due to cooperation between the multiple VEGFRs present in zebrafish. Analogous to this, there is a potential overlap in Amot signaling pathways as the closely related AMOTL-1 protein directly associates with Amot (M. Ernkvist, S. Audebert, P. Lecine, N. Luna-Persson, I. Sinha, M. Liu, A. Bratt, A. Horowitz, K. Aase, and L. Holmgren, in prep.) and overlaps with Amot in the expression pattern. This is supported by the finding that injection of murine amotl-1 mRNA can rescue (although with a delay as compared with amot mRNA) the ISV effect observed with antisense amot morpholinos. In addition to the ISV phenotype, we also observed that the $\mathrm{pHBC}$ and $\mathrm{pMBC}$ vessels were dilated at $36 \mathrm{hpf}$. This was reversible as the dilation was no longer detected at $60 \mathrm{hpf}$.

Genetic ablation of amot in mouse embryogenesis yielded a phenotype quite similar to that of $\mathrm{Amot}^{\mathrm{KD}}$ in zebrafish. Deletion of amot resulted in $\sim 75 \%$ lethality occurring between E11 and E11.5. Affected embryos suffered from insufficient capillary formation in the somitic region and defects in vascular integrity (dilation) in brain capillaries. A defect in cell migration and tube formation was suggested by the findings that Amot-deficient stem cells showed a severe impairment in the formation of vascular sprouts in the collagen assay. Furthermore, intraocular injection of amot siRNA in wild-type mice inhibited choroid vessel infiltration into the retina in the laser-induced CNV model. No obvious defects were detected in the surviving Amot-deficient mice, which were fertile and of similar size to wild-type littermates. However, defects in pathological angiogenesis cannot be excluded.

\section{Amot links polarization and migration}

The effect of Amot deficiency was also analyzed at a cellular level using ES-derived ECs or siRNA-mediated knockdown of Amot in BCE cells. Amot deficiency did not alter the VEGF-stimulated differentiation of ECs in vitro. Furthermore, Amot does not appear to affect VEGF-stimulated cell proliferation or affect cell survival. However, loss of Amot renders the cells unresponsive to VEGF- or bFGF-stimulated chemotaxis. An interesting aspect of this observation would be that Amot is required for the translation of a growth factor-mediated signaling into cell movement. Efficient chemotaxis re- sults from coordinated chemoattractant gradient sensing, cell polarization, and cellular motility. There is increasing evidence that polarity proteins such as Par-6, PKC, and Patj are not only involved in the establishment of baso-lateral polarity but are also important for frontrear polarity during migration (Shin et al. 2007). Timelapse analysis showed that wild-type cells polarize with a leading edge, whereas Amot-deficient cells extended protrusions in an unpolarized manner. Consistent with these findings, we could show that Amot $^{-}$PmT-ECs exhibited loss of polarization of the Golgi apparatus. Recent results indicate that Amot functions as a scaffold for polarity proteins as well as the GAP Rich-1 in epithelial cells (Wells et al. 2006). Rich-1 is also expressed in ECs, where it localizes to the leading edge of migrating cells. In Amot ${ }^{-}$PmT-ECs, Rich-1 failed to localize to lamellipodia (Fig. 7). The subcellular localization of Amot to lamellipodia and the ability to bind cell polarity proteins as well as GTPase-regulating proteins indicates a function in the local orchestration of GTPase activity required for cytoskeletal reorganization during migration.

In summary, we have shown that Amot plays a specific role in regulating vascular formation through endothelial migration and cell polarity. Further studies are necessary to understand more precisely how these signaling pathways are coupled. Finally, the findings suggest that Amot is an attractive molecule to specifically target endothelial migration without affecting differentiation and survival pathways.

\section{Materials and methods}

\section{Tissue culture}

AB-1 murine ES cells were grown on a layer of mitomycin Ctreated murine embryonic fibroblast (MEF) feeder cells. ES cells were grown in ES medium containing Dulbecco's modified Eagle's medium (DMEM; Sigma) supplemented with 10\% KSR knockout replacement serum (GIBCO), $1 \mathrm{mM}$ Na-pyruvate, 2 $\mathrm{mM}$ L-glutamine, $0.1 \mathrm{mMb}$-mercaptoethanol, $1 \%$ penicillinstreptomycin, $1 \times$ nonessential amino acids (Sigma), and 50 $\mathrm{U} / \mathrm{mL}$ ESGRO (Chemicon International). Cells were grown at $37^{\circ} \mathrm{C}$ with $6 \% \mathrm{CO}_{2}$. MEFs were grown in ES medium without ESGRO and treated with $10 \mu \mathrm{g} / \mathrm{mL}$ mitomycin C (Sigma) for 2 h. To induce differentiation, ESGRO was omitted from the medium, and 1200 cells were allowed to aggregate on the lid of a nonadherent tissue culture dish. The medium was supplemented with VEGF where indicated.

\section{Antibodies and reagents}

Peptides corresponding to the $24 \mathrm{C}$-terminal amino acids were used to generate the C-terminal antibodies against Amot (Levchenko et al. 2003). Biotinylated isolectin-B4 (Sigma) was used to visualize the endothelium. Alexa Fluor 594-conjugated Phalloidin (Molecular Probes) was used to visualize actin. The Golgi apparatus was visualized using an antibody against the Golgi-specific protein p115 (BD Pharmingen; a kind gift from E. Raschberger, Ludwig Institute for Cancer Research [LICR], 
Stockholm, Sweden). Other antibodies used were von Willenbrant factor (vWF; DAKO), PECAM (BD Pharmingen), FITCmouse monoclonal anti- $\alpha$ smooth muscle actin antibody $(\mathrm{R} \& \mathrm{D})$, paxillin (BD Transduction Laboratories), VEGFR2 (BD Transduction Laboratories), and $\beta$-actin (Sigma). The secondary antibodies were anti-rabbit HRP (GE Healthcare), Alexa Fluor antibodies (Molecular Probes), and anti-rabbit FITC (DAKO). The nucleus was visualized using mounting medium containing DAPI (Vector Laboratories). VEGF was purchased from Peprotec Ltd.

\section{Zebrafish analysis}

Adult zebrafish were grown in the fish facility with a 14-h light/ 12-h dark cycle. Zebrafish embryos harboring the Tg (fli: EGFP ${ }^{y 1}$ transgene (Lawson and Weinstein 2002) were injected at the one- to four-cell stage with morpholinos and raised at $28^{\circ} \mathrm{C}$ in standard E3 water supplemented with $0.003 \%$ PTU (phenyl-2-thiourea). BLAST searches on Ensembl were done to identify an amot zebrafish ortholog. The following morpholinos purchased from GeneTools LLC were used to inactivate the zebrafish amot: 5' -CCACTGACACAACTACCACCAAGTG-3' (Amot exon 2) and 5'-CCTTACTTGACCTATTGAGGAGCA G-3' (Amot exon 3). A mismatch morpholino, 5'-CCACTcA CACAAgTACgACgAAcTG-3' (Amot exon 2 ctrl), was used as a negative control. All morpholinos were injected at $250 \mu \mathrm{M}$ diluted in $0.3 \times$ Danieau's solution with $0.05 \%$ phenol red included as a tracer. Human p80-Amot and mouse AMOTL1 mRNAs were synthesized from linearized templates in vitro using Ambion's Message Machine kits. For the rescue experiments, mRNAs were mixed with amot morpholinos and in jected at $\sim 100 \mathrm{pg}$ per embryo. RT-PCR was used to confirm knockdown, using the primers 5 '-ACTCGATGGTCCCACA CATT-3' and 5'-GAATGACCCATTGGTGGAAG-3'. Phenotypic consequences of morpholino gene knockdown were assessed from 30 to $60 \mathrm{hpf}$ on a Leica MZ16 stereomicroscope equipped with epifluoresence. Confocal images of vascular phenotypes were taken on a Zeiss Axiovert 200M microscope using a $20 \times$ Apochromat objective.

\section{In situ hybridization and PECAM whole-mount staining}

Nonradioactive whole-mount hybridization was carried out as described (Bostrom et al. 1996). Two different probes were used to confirm staining. First, a 679-base-pair (bp) fragment from mouse Amot cDNA was amplified using PCR and primers sense, $\left(5^{\prime}-3^{\prime}\right)$ GAGCTCCTCCGGGAGAAG, and reverse, (5'$\left.3^{\prime}\right)$ TGGACCAGCCATTGGAGC. The purified fragment was cloned into the PCR-vector pTAdv (Clontech) and then subcloned into pSPT18 and pSPT19 (Roche) to generate antisense and sense probes. The second probe $(600 \mathrm{bp})$ was generated using a PCR probe containing the T7 sequence $\left(5^{\prime}-3^{\prime}\right)$ TGATTAAT ACGATCACTATAGG at one end. The primers used were sense $\left(5^{\prime}-3^{\prime}\right)$, TGGCTACTAGTGGAGTTAAAG, and reverse $\left(5^{\prime}-3^{\prime}\right)$, CTGGCTGCTGCTGCGCTG. The specificity to Amot was confirmed by BLAST search. PECAM whole-mount staining was performed on embryos dissected between E9.5 and E11 as previously described (Levchenko et al. 2003).

Immunofluorescence of cells, sections, and embryoid bodies

Cells: Cultured cells were plated in chamber slides and allowed to grow and adhere. The cells were fixed in 4\% PFA for $10 \mathrm{~min}$ at room temperature and permeabilized in $0.1 \%$ Triton X-100 (Sigma) for $1 \mathrm{~min}$. Nonspecific reactivity was blocked by incubating with $5 \%$ horse serum in PBS for $1 \mathrm{~h}$ before addition of primary antibody in blocking buffer for $1 \mathrm{~h}$. Antibody binding was detected with fluorescent-labeled secondary antibodies.

Sections: Embryos were fixed in 4\% paraformaldehyde (PFA) overnight, dehydrated, and embedded in paraffin. Five-micron sections were deparaffinized, rehydrated, and boiled for $10 \mathrm{~min}$ in citric acid $(1.8 \mathrm{mM}$ sodium citrate, $8.2 \mathrm{mM}$ citric acid at $\mathrm{pH}$ 6.0) to unmask the antigen. After washing in PBS for 5 min, the anti-Amot antibody and isolectin-B4 were added, and the sections were incubated overnight at room temperature. Antibody binding was detected with fluorescent-labeled secondary antibodies, and isolectin was detected using the fluorescent streptavidin kit (Vector laboratories Inc.).

Embryoid bodies: Embryoid bodies were stained as described (Jakobsson et al. 2006).

\section{Mouse breeding and genotyping}

The Amot-deficient strain has been described (Shimono and Behringer 2003). Mice and embryos used in this study have been backcrossed up to six generations into the C57/B6 strain. Mouse tail tips, yolk sacs, or embryos were used for genotyping by PCR as described (Shimono and Behringer 2003).

\section{Western blot}

Cell lysates were analyzed by SDS-PAGE, and proteins were transferred to nitrocellulose membrane. Nonspecific binding was blocked for $1 \mathrm{~h}$ in $10 \%$ dried milk in PBS containing $0.1 \%$ Tween (PBS-T). The filter was incubated overnight at $4{ }^{\circ} \mathrm{C}$ in $5 \%$ dried milk in PBS-T with primary antibody. The secondary antibody (anti-rabbit HRP, anti-mouse HRP, or anti-rat HRP) was diluted 1:10,000 (GE Healthcare) and incubated for $1 \mathrm{~h}$ at room temperature. Signal was detected using the luminol reagent from Santa Cruz Biotechnology.

\section{Laser-induced CNV}

CNV was generated by krypton laser-induced rupture of Bruch's membrane, and eyes were enucleated after $10 \mathrm{~d}$, as previously described (Berglin et al. 2003). Mice received an intravitreal injection of $20 \mathrm{pmol}(1 \mu \mathrm{L}$ ) of Amot siRNA (smart pool from Dharmacon) immediately after laser treatment at 0,3 , and $6 \mathrm{~d}$. Ten mice were treated in each group in two independent experiments. The vascularization of each lesion was estimated from the PECAM staining by quantifying the number of PECAM-positive pixels per plaque, and data from each lesion were treated as a single statistical point.

\section{Immortalization of ECs derived from embryoid bodies}

Immortalization of ECs derived from embryoid bodies was performed as described (Balconi et al. 2000). Briefly, 11-d-old embryoid bodies were collected and disaggregated by $1.5 \mathrm{mg} / \mathrm{mL}$ collagenase A (Sigma). After disaggregating, $25 \mu \mathrm{g} / \mathrm{mL}$ DNase was added. Cells were allowed to grow for $2 \mathrm{~d}$ before infection with PmT virus harvested from GgP + E cells (kindly provided by Dr. Elisabeta Dejana, Mario Negri Institute for Pharmacological Research, Milan, Italy). Wild-type ECs were selected with $800 \mu \mathrm{g} / \mathrm{mL}$ G418 (Sigma).

\section{Real-time PCR}

Total RNA was extracted from 8-d-old embryoid bodies and PmT-ECs using the RNeasy minikit (Qiagen). Contaminating genomic DNA was removed with DNase (Qiagen), and $3 \mu \mathrm{g}$ of total RNA was used for first-strand cDNA synthesis using oligo 
dT primers and the SuperScript III First-Strand kit (Invitrogen). The reaction was performed in duplicate with the 7500 realtime PCR system (Applied Biosystems). The expression levels of the EC-specific genes in Amot ${ }^{-}$embryoid bodies and PmT-ECs were normalized against actin and compared with the expression level of wild-type bodies and cells. A ratio of 1 corresponds to an equal expression level of wild-type and Amot $^{-}$cells. The primers used were $\beta$-actin sense, $\left(5^{\prime}-3^{\prime}\right)$ CACTATTGGCAAC GAGCGG; $\beta$-actin antisense, $\left(5^{\prime}-3^{\prime}\right)$ TCCATACCCAAG AAGGC; PECAM sense, $\left(5^{\prime}-3^{\prime}\right)$ TACTGCAGGCATCGGCA AA; PECAM antisense, $\left(5^{\prime}-3^{\prime}\right)$ GCATTTCGCACACCTGGAT; VEGFR-1 sense, (5'-3') GGGCAGACTCTTGTCCTCAACT; VEGFR-1 antisense, (5'-3') CAGCTCATTTGCACCCTCGT; VEGFR-2 sense, $\left(5^{\prime}-3^{\prime}\right)$ TACAGACCCGGCCAAACAA; VEGFR-2 antisense, (5'-3') TTTCCCCCCTGGAAATCCT; $V E$-cadherin sense, $\left(5^{\prime}-3^{\prime}\right)$ AGGACAGCAACTTCACCCTCA; $V E$-cadherin antisense, $\left(5^{\prime}-3^{\prime}\right)$ AACTGCCCATACTTGACC GTG; $N P-1$ sense, $\left(5^{\prime}-3^{\prime}\right)$ TCCGCAGCGACAAATGTG; $N P-1$ antisense, $\left(5^{\prime}-3^{\prime}\right)$ GGTAACCGGGAGATGTGAGGT.

\section{Focal adhesion measurements}

For focal adhesion measurements, the cells were stained with the paxillin-specific antibody, photographed, and analyzed using the program Lucia G. Threshold values were set between 0.2 and $20 \mu \mathrm{m}$ to exclude background.

\section{Cell migration assays}

The Boyden chamber migration assay was performed as described (Levchenko et al. 2003). Wound healing was performed as described (Ernkvist et al. 2006).

\section{Proliferation assay}

Fifty-thousand cells were seeded onto $25-\mathrm{mm}$ plates and starved overnight in $0.5 \%$ FCS. VEGF (50 ng/mL) was added, and cells were counted every day. The assay was done in triplicate in three independent experiments.

\section{Time lapse}

Cells were seeded on $3-\mathrm{cm}$ cell plates, and images were collected every $6 \mathrm{~min}$. The images were used to create a movie using the Ulead GIF Animator 5, where every second corresponds to 10 frames.

\section{Polarization analysis}

Cells were plated subconfluently on chamber slides and allowed to attach and spread for $5 \mathrm{~h}$. Thereafter, the cells were fixed and stained for the Golgi apparatus, actin fibers, and the nucleus as described above. If the Golgi apparatus was located within a $120^{\circ}$ sector, the cell was defined as polarized, and if it was located within more than a $120^{\circ}$ sector, it was defined as unpolarized.

\section{Rac pull-down assay}

Cells were serum-starved overnight and then stimulated by growth medium containing 10\% FBS for $30 \mathrm{~min}$. After the treatment, cells were harvested, washed with PBS, and lysed in $1 \times$ $\mathrm{Mg}^{2+}$ Lysis Buffer (125 mM HEPES at $\mathrm{pH} 7.5,750 \mathrm{mM} \mathrm{NaCl}, 5 \%$ Igepal CA-630, $50 \mathrm{mM} \mathrm{MgCl}$, $5 \mathrm{mM}$ EDTA, 10\% glycerol). Clarified lysates were incubated with PAK-1 PBD-agarose (Upstate Biotechnology) for $90 \mathrm{~min}$ at $4^{\circ} \mathrm{C}$ and washed three times with $1 \times \mathrm{Mg}^{2+}$ Lysis Buffer. Rac1 was detected by immunoblot analysis.

\section{Amot knockdown in cell lines}

For silencing endogenous Amot in $293 \mathrm{~T}$ cells, a 19-nucleotide sequence corresponding to bases 2806-2824 of Homo sapiens amot mRNA (NM_133265.2) was selected as the target. A small hairpin RNA (shRNA) incorporating this sequence was cloned into pLenti-lox3.7 (pLL3.7) as described previously (Rubinson et al. 2003). The resulting vector was transfected together with packaging vectors VSVG and D8.9 into $293 \mathrm{~T}$ cells, and viral supernatant was collected after 48 and $72 \mathrm{~h}$. This supernatant was then used to infect fresh $293 \mathrm{~T}$ cells, and GFP-positive infected cells were selected by flow cytometry. For silencing Amot in BCE cells, a smart pool from Dharmacon was used and transfected according to the manufacturer's instructions. Western blot analysis was used to confirm knockdown of both isoforms of Amot.

\section{Statistical analyses}

The statistical significance was determined using the Student's $t$-test, where $P<0.001\left(^{\star \star \star}\right), P<0.01\left(^{\star \star}\right)$, and $P<0.05\left(^{\star}\right)$.

\section{Acknowledgments}

We thank the Zebrafish International Resource Center (ZIRC) for fish; S. Warner, S. Kisana, and U. Wargh in the Karolinska Institute zebrafish facility; and C. Betsholtz for use of his equipment. We also thank E. Dejana (Mario Negri Institute for Pharmacological Research, Milan, Italy) for generously providing the PmT-virus and E. Raschberger (LICR, Stockholm Branch, Sweden) for the Golgi-specific antibody. We also thank $\AA$. Mackenzie (BMC, Uppsala University, Sweden) and G. Balconi (Mario Negri Institute for Pharmacological Research, Milan, Italy) for expert advice, and J. Dixelius (Karolinska Institutet, Stockholm, Sweden) for expert assistance during paxillin measurements. This work was supported by grants from the Swedish Cancer Society, Cancer Society of Stockholm, Swedish Research Council, and grants from the Karolinska Institutet, Stockholm.

\section{References}

Aepfelbacher, M., Essler, M., Huber, E., Sugai, M., and Weber, P.C. 1997. Bacterial toxins block endothelial wound repair. Evidence that Rho GTPases control cytoskeletal rearrangements in migrating endothelial cells. Arterioscler. Thromb. Vasc. Biol. 17: 1623-1629.

Balconi, G., Spagnuolo, R., and Dejana, E. 2000. Development of endothelial cell lines from embryonic stem cells: A tool for studying genetically manipulated endothelial cells in vitro. Arterioscler. Thromb. Vasc. Biol. 20: 1443-1451.

Berglin, L., Sarman, S., van der Ploeg, I., Steen, B., Ming, Y., Itohara, S., Seregard, S., and Kvanta, A. 2003. Reduced choroidal neovascular membrane formation in matrix metalloproteinase-2-deficient mice. Invest. Ophthalmol. Vis. Sci. 44: 403-408.

Bostrom, H., Willetts, K., Pekny, M., Leveen, P., Lindahl, P., Hedstrand, H., Pekna, M., Hellstrom, M., Gebre-Medhin, S., Schalling, M., et al. 1996. PDGF-A signaling is a critical event in lung alveolar myofibroblast development and alveogenesis. Cell 85: 863-873.

Bratt, A., Wilson, W.J., Troyanovsky, B., Aase, K., Kessler, R., Van Meir, E.G., and Holmgren, L. 2002. Angiomotin belongs to a novel protein family with conserved coiled-coil and PDZ 
binding domains. Gene 298: 69-77.

Bratt, A., Birot, O., Sinha, I., Veitonmaki, N., Aase, K., Ernkvist, M., and Holmgren, L. 2005. Angiomotin regulates endothelial cell-cell junctions and cell motility. J. Biol. Chem. 280: 34859-34869.

Carmeliet, P., Ferreira, V., Breier, G., Pollefeyt, S., Kieckens, L., Gertsenstein, M., Fahrig, M., Vandenhoeck, A., Harpal, K., Eberhardt, C., et al. 1996. Abnormal blood vessel development and lethality in embryos lacking a single VEGF allele. Nature 380: 435-439.

Childs, S., Chen, J.N., Garrity, D.M., and Fishman, M.C. 2002. Patterning of angiogenesis in the zebrafish embryo. Development 129: 973-982.

Covassin, L.D., Villefranc, J.A., Kacergis, M.C., Weinstein, B.M., and Lawson, N.D. 2006. Distinct genetic interactions between multiple Vegf receptors are required for development of different blood vessel types in zebrafish. Proc. Natl. Acad. Sci. 103: 6554-6559.

Ernkvist, M., Aase, K., Ukomadu, C., Wohlschlegel, J., Blackman, R., Veitonmaki, N., Bratt, A., Dutta, A., and Holmgren, L. 2006. p130-angiomotin associates to actin and controls endothelial cell shape. FEBS J. 273: 2000-2011.

Ferrara, N., Carver-Moore, K., Chen, H., Dowd, M., Lu, L., O'Shea, K.S., Powell-Braxton, L., Hillan, K.J., and Moore, M.W. 1996. Heterozygous embryonic lethality induced by targeted inactivation of the VEGF gene. Nature 380: 439-442.

Franca-Koh, J. and Devreotes, P.N. 2004. Moving forward: Mechanisms of chemoattractant gradient sensing. Physiology (Bethesda) 19: 300-308.

Gerhardt, H., Golding, M., Fruttiger, M., Ruhrberg, C., Lundkvist, A., Abramsson, A., Jeltsch, M., Mitchell, C., Alitalo, K., Shima, D., et al. 2003. VEGF guides angiogenic sprouting utilizing endothelial tip cell filopodia. J. Cell Biol. 161: 1163-1177.

Gluzman-Poltorak, Z., Cohen, T., Herzog, Y., and Neufeld, G. 2000. Neuropilin-2 is a receptor for the vascular endothelial growth factor (VEGF) forms VEGF-145 and VEGF-165 [corrected]. J. Biol. Chem. 275: 18040-18045.

Goishi, K. and Klagsbrun, M. 2004. Vascular endothelial growth factor and its receptors in embryonic zebrafish blood vessel development. Curr. Top. Dev. Biol. 62: 127-152.

Holmgren, L., Ambrosino, E., Birot, O., Tullus, C., Veitonmaki, N., Levchenko, T., Carlson, L.M., Musiani, P., Iezzi, M., Curcio, C., et al. 2006. A DNA vaccine targeting angiomotin inhibits angiogenesis and suppresses tumor growth. Proc. Nat1. Acad. Sci. 103: 9208-9213.

Hynes, R.O. 2002. A reevaluation of integrins as regulators of angiogenesis. Nat. Med. 8: 918-921.

Isogai, S., Lawson, N.D., Torrealday, S., Horiguchi, M., and Weinstein, B.M. 2003. Angiogenic network formation in the developing vertebrate trunk. Development 130: 5281-5290.

Jakobsson, L., Kreuger, J., Holmborn, K., Lundin, L., Eriksson, I., Kjellen, L., and Claesson-Welsh, L. 2006. Heparan sulfate in trans potentiates VEGFR-mediated angiogenesis. Dev. Cell 10: 625-634.

Jones, E.A., le Noble, F., and Eichmann, A. 2006. What determines blood vessel structure? Genetic prespecification vs. hemodynamics. Physiology (Bethesda) 21: 388-395.

Kvanta, A. 2006. Ocular angiogenesis: The role of growth factors. Acta Ophtalmol. Scand. 84: 282-288.

Lawson, N.D. and Weinstein, B.M. 2002. In vivo imaging of embryonic vascular development using transgenic zebrafish. Dev. Biol. 248: 307-318.

Leslie, J.D., Ariza-McNaughton, L., Bermange, A.L., McAdow, R., Johnson, S.L., and Lewis, J. 2007. Endothelial signalling by the Notch ligand Delta-like 4 restricts angiogenesis. De- velopment 134: 839-844.

Levchenko, T., Aase, K., Troyanovsky, B., Bratt, A., and Holmgren, L. 2003. Loss of responsiveness to chemotactic factors by deletion of the C-terminal protein interaction site of angiomotin. J. Cell Sci. 116: 3803-3810.

Levchenko, T., Bratt, A., Arbiser, J.L., and Holmgren, L. 2004. Angiomotin expression promotes hemangioendothelioma invasion. Oncogene 23: 1469-1473.

Nobes, C.D. and Hall, A. 1995. Rho, rac, and cdc42 GTPases regulate the assembly of multimolecular focal complexes associated with actin stress fibers, lamellipodia, and filopodia. Cell 81: 53-62.

Nobes, C.D. and Hall, A. 1999. Rho GTPases control polarity, protrusion, and adhesion during cell movement. J. Cell Biol. 144: $1235-1244$

Olsson, A.K., Dimberg, A., Kreuger, J., and Claesson-Welsh, L. 2006. VEGF receptor signalling-In control of vascular function. Nat. Rev. Mol. Cell Biol. 7: 359-371.

Ong, S.H., Dilworth, S., Hauck-Schmalenberger, I., Pawson, T., and Kiefer, F. 2001. ShcA and Grb2 mediate polyoma middle $\mathrm{T}$ antigen-induced endothelial transformation and Gab1 tyrosine phosphorylation. EMBO J. 20: 6327-6336.

Richnau, N. and Aspenstrom, P. 2001. Rich, a rho GTPase-activating protein domain-containing protein involved in signaling by Cdc42 and Rac1. J. Biol. Chem. 276: 35060-35070.

Risau, W. 1997. Mechanisms of angiogenesis. Nature 386: 671674.

Rubinson, D.A., Dillon, C.P., Kwiatkowski, A.V., Sievers, C., Yang, L., Kopinja, J., Rooney, D.L., Ihrig, M.M., McManus, M.T., Gertler, F.B., et al. 2003. A lentivirus-based system to functionally silence genes in primary mammalian cells, stem cells and transgenic mice by RNA interference. Nat. Genet. 33: 401-406.

Shimono, A. and Behringer, R.R. 2003. Angiomotin regulates visceral endoderm movements during mouse embryogenesis. Curr. Biol. 13: 613-617.

Shin, K., Wang, Q., and Margolis, B. 2007. PATJ regulates directional migration of mammalian epithelial cells. EMBO Rep. 8: $158-164$.

Singer, S.J. and Kupfer, A. 1986. The directed migration of eukaryotic cells. Annu. Rev. Cell Biol. 2: 337-365.

Soker, S., Takashima, S., Miao, H.Q., Neufeld, G., and Klagsbrun, M. 1998. Neuropilin-1 is expressed by endothelial and tumor cells as an isoform-specific receptor for vascular endothelial growth factor. Cell 92: 735-745.

Troyanovsky, B., Levchenko, T., Mansson, G., Matvijenko, O., and Holmgren, L. 2001. Angiomotin: An angiostatin binding protein that regulates endothelial cell migration and tube formation. J. Cell Biol. 152: 1247-1254.

Vittet, D., Prandini, M.H., Berthier, R., Schweitzer, A., MartinSisteron, H., Uzan, G., and Dejana, E. 1996. Embryonic stem cells differentiate in vitro to endothelial cells through successive maturation steps. Blood 88: 3424-3431.

Weinstein, B.M. 2002. Plumbing the mysteries of vascular development using the zebrafish. Semin. Cell Dev. Biol. 13: $515-522$.

Wells, C.D., Fawcett, J.P., Traweger, A., Yamanaka, Y., Goudreault, M., Elder, K., Kulkarni, S., Gish, G., Virag, C., Lim, C., et al. 2006. A Rich1/Amot complex regulates the Cdc42 GTPase and apical-polarity proteins in epithelial cells. Cell 125: 535-548.

Yancopoulos, G.D., Klagsbrun, M., and Folkman, J. 1998. Vasculogenesis, angiogenesis, and growth factors: Ephrins enter the fray at the border. Cell 93: 661-664. 


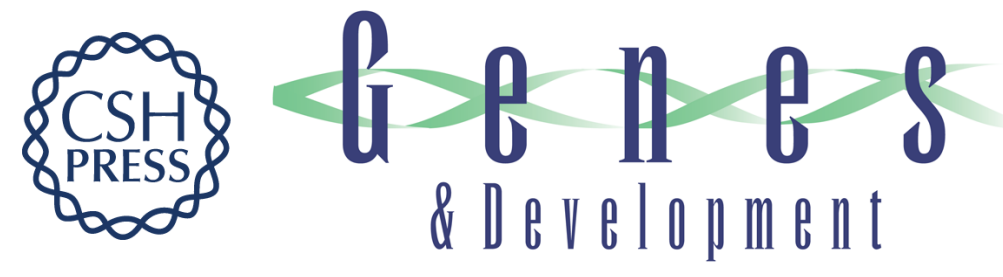

\section{Angiomotin regulates endothelial cell migration during embryonic angiogenesis}

Karin Aase, Mira Ernkvist, Lwaki Ebarasi, et al.

Genes Dev. 2007, 21:

Access the most recent version at doi:10.1101/gad.432007

Supplemental http://genesdev.cshlp.org/content/suppl/2007/08/06/21.16.2055.DC1
Material

References This article cites 41 articles, 16 of which can be accessed free at: http://genesdev.cshlp.org/content/21/16/2055.full.html\#ref-list-1

\section{License}

Email Alerting

Service

Receive free email alerts when new articles cite this article - sign up in the box at the top right corner of the article or click here.

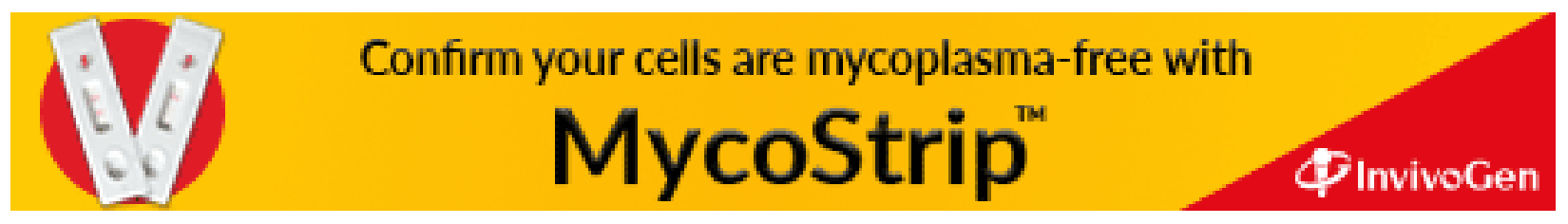

